PROCEEDINGS OF THE

AMERICAN MATHEMATICAL SOCIETY

Volume 136, Number 12, December 2008, Pages 4373-4383

S 0002-9939(08)09520-8

Article electronically published on July 8, 2008

\title{
MULTITOWERS, CONJUGACIES AND CODES: THREE THEOREMS IN ERGODIC THEORY, ONE VARIATION ON ROKHLIN'S LEMMA
}

\author{
S. ALPERN AND V. S. PRASAD
}

(Communicated by Jane M. Hawkins)

We dedicate this paper to the memory of Shizuo Kakutani. We miss his kind manner, gentle presence and keen insight.

\begin{abstract}
We show that three theorems about the measurable dynamics of a fixed aperiodic measure preserving transformation $\tau$ of a Lebesgue probability space $(X, \mathcal{A}, \mu)$ are equivalent. One theorem asserts that the conjugates of $\tau$ are dense in the uniform topology on the space of automorphisms. The other two results assert the existence of a partition of the space $X$ with special properties. One partition result shows that given a mixing Markov chain, there is a code (i.e., a partition of the space) so that the itinerary process given by $\tau$ and the partition has the distribution of the given Markov Chain. The other partition result is a generalization of the Rokhlin Lemma, stating that the space can be partitioned into denumerably many columns and the measures of the columns can be prescribed in advance. Thus the first two results are equivalent to this strengthening of Rokhlin's Lemma.
\end{abstract}

\section{INTRODUCTION}

We show that three (apparently different) basic theorems about the measurable dynamics of a fixed aperiodic invertible measure preserving transformation (an automorphism) $\tau$ of a Lebesgue space $(X, \mathcal{A}, \mu)$ are simple corollaries of each other. In this sense, the theorems are equivalent. Two theorems assert the existence of a partition of $X$ with special properties under the action of $\tau$, and the third theorem states that the conjugates of $\tau$ form a dense class in the space of automorphisms in a certain topology on the automorphisms:

- MRT: The Multiple Rokhlin Tower theorem for $\tau$ provides a simple condition for the existence of a partition of the space $X$ into denumerably many columns for $\tau$, with prescribed measures. MRT is a generalization of one of the basic constructions in ergodic theory, Rokhlin's Lemma (see for example Kornfeld's survey [18]).

- CMC: The Coding theorem shows that given any mixing Markov chain $\mathbf{P}$, there is a partition of the space $X$ so that $\tau$ moves the partition elements according to the transitions prescribed by the Markov chain $\mathbf{P}$. CMC is a

Received by the editors November 13, 2007.

2000 Mathematics Subject Classification. Primary 37A05; Secondary 60J10.

Key words and phrases. Rokhlin towers, conjugacy, coding, stationary aperiodic irreducible Markov chain.

(C)2008 American Mathematical Society 4373

Reverts to public domain 28 years from publication 
reformulation and strengthening of a coding question of Kieffer [17] which asks if a stationary stochastic process can be coded to have prescribed marginal distributions.

- ACT: The Approximate Conjugacy Theorem for $\tau$ states that given any totally ergodic automorphism, then there is some conjugate of $\tau$ that agrees pointwise with the totally ergodic automorphism, except possibly on some previously prescribed set of small measure. ACT generalizes Halmos's Conjugacy Lemma [13.

In Theorem 3.1 we give a short proof that CMC implies MRT. Combining this new result with the known implications $\mathbf{M R T} \Rightarrow \mathbf{A C T} \Rightarrow \mathbf{C M C}$ (these implications are in Appendix A of our book [5, which also contains detailed references to our papers where these results were originally proved), immediately proves Corollary 3.2 the equivalence of the three theorems. To make this paper self-contained, we provide another proof of the equivalence of the three theorems, Corollary 3.2 . by giving proofs of the following chain of implications: $\mathbf{M R T} \Rightarrow \mathbf{C M C} \Rightarrow \mathbf{A C T} \Rightarrow$ MRT. The proofs of the last two implications have not been previously published; a proof of the implication MRT $\Rightarrow \mathbf{C M C}$ follows from the chain of implications in Appendix A of our book Typical dynamics of volume preserving homeomorphisms 5]. Appendix A of our book also includes a complete proof of MRT, the variation on Rokhlin's Lemma.

In the next section we give complete statements of each of the three basic theorems on the dynamics of an aperiodic automorphism. Recent applications of each of these theorems in different contexts follow each statement. The equivalence of these apparently different theorems should be of interest to dynamicists and probabilists.

\section{Three theorems, MANy uses, A LitTle history}

Suppose $(X, \mathcal{A}, \mu)$ is a (nonatomic) Lebesgue probability space and $\tau$ an invertible measure preserving transformation of $(X, \mathcal{A}, \mu)$ onto itself (we say $\tau$ is an automorphism of $X$ ) which is aperiodic (the periodic points for $\tau$ form a $\mu$-null set). Although all theorems are stated for aperiodic automorphisms, there is no loss of generality if the reader assumes that $\tau$ is ergodic (an aperiodic automorphism always has a decomposition into ergodic components) and that the measure space is the unit interval with length measure (since every Lebesgue space is conjugate by a measure preserving isomorphism to the unit interval with length measure). Furthermore, all statements about sets and automorphisms are true if they hold except possibly on a set of measure zero.

2.1. MultiTowers. Alpern proved a generalization of Rokhlin's Lemma that we call the Multiple Rokhlin Tower theorem and refer to as MRT. A proof of this theorem is in our book [5].

Theorem 2.1 (MRT). Let $\tau$ be an aperiodic $\mu$-preserving automorphism of a Lebesgue probability space $(X, \mathcal{A}, \mu)$ onto itself. Let $\pi=\left(\pi_{1}, \pi_{2}, \ldots\right)$ be a probability distribution such that $\left\{k: \pi_{k}>0\right\}$ is a relatively prime set of positive integers (we call such a distribution $\pi$, an aperiodic distribution). Then there is a partition $\mathcal{P}=\left\{P_{k, i}: k=1,2, \ldots ; i=1,2, \ldots, k\right\}$ of $X$ so that for each integer $k=1,2, \ldots$,

(1) $P_{k, i}=\tau^{i-1}\left(P_{k, 1}\right)$ for $i=1,2, \ldots, k$,

(2) $\mu\left(\bigcup_{i=1}^{k} P_{k, i}\right)=\pi_{k}$.

We call $\mathcal{P}$ a MultiTower partition for $\tau$ with aperiodic distribution $\pi$. 
The Rokhlin Lemma asserts that for $\tau$ as above, $n$ any positive integer and $\epsilon$ any positive number less than 1 , there is a subset $R \in \mathcal{A}$, so that $R$ and the first $n-1$ iterates of $R$ are disjoint and $\mu\left(\bigcup_{i=0}^{n-1} \tau^{i}(R)\right)=1-\epsilon$. The Multiple Rokhlin Tower theorem when applied to the probability vector $\pi$ with $\pi_{1}=\epsilon$ and $\pi_{n}=1-\epsilon$ yields a set $P_{n, 1}$, which has the property of the Rokhlin set $R$ in Rokhlin's Lemma. We note that the Multiple Rokhlin Tower theorem states more generally (than the Rokhlin Lemma) that an aperiodic automorphism has a representation by (denumerably many) columns $\left(\bigcup_{i=1}^{k} P_{k, i}\right)$ of given heights $(k)$ and given measures $\left(\pi_{k}\right)$ as long as the heights are relatively prime (i.e., $\pi$ is an aperiodic distribution). Furthermore, the MRT asserts the existence of a set $P=\bigcup_{k} P_{k, 1}$ with relative distribution of first return times to $P$ given by $\pi$. Note that if $\pi$ is not aperiodic and $d>1$ is a common multiple of all $k$ 's for which $\pi_{k}>0$, then for any set $P$ whose relative distribution of return times to $P$ is given by $\pi$ and for any $m$ which is not a multiple of $d$, we would have $\mu\left(P \cap \tau^{m}(P)\right)=0$. Such a set $P$ cannot exist for every aperiodic automorphism $\tau$ (for example if $\tau$ is mixing).

MRT is a discrete analog of D. Rudolph's flow theorem [23] that every ergodic aperiodic flow on $X$ has a representation as a flow built under a function $f$ taking only two values $p$ and $q$, as long as $p / q$ is irrational; furthermore, Krengel showed that one can choose the representation to achieve the distribution $\mu\{f=p\}=$ $\rho \mu\{f=q\}$ for any $0<\rho<\infty$. In other words, the flow on $X$ has a Poincaré cross section $X_{0}$ with first return times exactly $p$ and $q$ of the prescribed distribution.

For finite dimensional aperiodic distributions $\pi$, Grillenberger and Krengel [1] previously considered a deep generalization of Theorem 2.1 where they seek a finite dimensional MultiTower partition of $X$ that also generates the sigma algebra. Extensions of MRT to automorphisms of an infinite measure space are in [3] and [9]. Versions of the MRT for an aperiodic nonsingular automorphism $\tau$ of a Lebesgue space are in [4. Prikhod'ko proved an extension of Theorem 2.1 for $\mathbb{Z}^{d}$-actions 22. Recently using methods different from Prikhod'ko, A. Sahin also proved a $\mathbb{Z}^{d}$-analog of MRT [25.

MRT is a basic result in studying measure preserving homeomorphisms of manifolds. Alpern and Prasad use MRT to prove that any measure theoretic property that is generic for automorphisms of a Lebesgue measure space is also a generic property for measure preserving homeomorphisms of manifolds. The latter result and its extensions form one of the main topics in our book Typical dynamics of volume preserving homeomorphisms [5]. In recent work on topological (and measurable) orbit equivalence, Kornfeld and Ormes [19] use MRT to prove strong orbit equivalence theorems and generalizations of the Jewett-Krieger theorem. A nice survey of the use of MRT and associated Bratteli diagrams to obtain Vershik adic-maps in topological orbit equivalence theory is in 18 .

2.2. Coding. The second of our three basic theorems shows that it is always possible to represent the transition probabilities of a mixing Markov Chain on a discrete state space using any aperiodic automorphism $\tau$ and some partition of the space. A mixing Markov chain on a discrete space is one for which the transition probability matrix $\mathbf{P}$ gives rise to a positive recurrent, irreducible and aperiodic Markov chain on some subset of the natural numbers $\mathbb{N}$; for us, a code on $X$ is a measurable partition of $X$, indexed by the state space of the Markov Chain. We refer to this property of any antiperiodic automorphism $\tau$ to Code Markov Chains as CMC. 
Theorem 2.2 (CMC). Let $\tau$ be an aperiodic $\mu$-preserving automorphism of a Lebesgue probability space $(X, \mathcal{A}, \mu)$ onto itself. Suppose that $\mathbf{P}=(p(i, j): i, j \in \mathbb{N})$ is the transition probability matrix for a positive recurrent, aperiodic, irreducible Markov chain with denumerable state space $\mathbb{N}$, the set of nonnegative integers. Let $\mathbf{r}=(r(i): i \in \mathbb{N})$ be the unique positive invariant distribution (i.e., $\mathbf{r} \mathbf{P}=\mathbf{r}$, so $\left.r(j)=\sum_{i \in \mathbb{N}} r(i) p(i, j)\right)$. Then there is a partition $\mathcal{R}=\left\{R_{i}: i \in \mathbb{N}\right\}$ of $X$ such that for all $i, j \in \mathbb{N}$,

$$
\mu\left(R_{i} \cap \tau^{-1} R_{j}\right)=\mu\left(R_{i}\right) p(i, j)=r(i) p(i, j) .
$$

This result extends to denumerable state spaces, J. Kieffer's finite state space result [17] where he considered the question of coding a stationary stochastic process, to one with prescribed marginal distributions on a finite state space. After reformulation, Kieffer notes that Theorem 2.2 for finite state space Markov Chains and ergodic $\tau$ follows from the deep result of Grillenberger and Krengel (mentioned in section 2.1) 11]. Cohen [8] considers the following finite dimensional variant of Theorem 2.2 the so-called rotational representations of $\mathbf{P}$ : Given an $n \times n$ stochastic matrix $(p(i, j): 1 \leq i, j \leq n)$, find a circle rotation $\tau$ and a circle partition $\mathcal{P}$ consisting of intervals satisfying (2.1). See also the work of Alpern [2], Haigh [12, Kalpazidou [15], Kalpazidou-Tzouvaras [14] and Alpern-Prasad 6] for related developments on rotational representations of stochastic matrices and cycle decompositions of Markov chains.

For $d$-dimensional actions, Pivato [21] has an analog of CMC.

2.3. Conjugacy. If $\tau$ and $\sigma$ are automorphisms of $(X, \mathcal{A}, \mu)$ and $(Y, \mathcal{B}, \nu)$ respectively, then they are conjugate if there is an invertible measure preserving bijection $\psi:(X, \mathcal{A}, \mu) \rightarrow(Y, \mathcal{B}, \nu)$ so that $\psi \tau \psi^{-1}(y)=\sigma(y)$ for almost all $y \in Y$. A fundamental problem in ergodic theory is to determine when two automorphisms are conjugate. The Approximate Conjugacy Theorem, referred to as ACT, addresses the question of whether it's possible to find some conjugate of $\tau$ which agrees with $\sigma$ on most of the space $Y$ when $\sigma$ is a totally ergodic automorphism (an automorphism is totally ergodic if all of its periodic sets are trivial, i.e. of full or zero measure).

Theorem 2.3 (ACT). Let $\tau$ be an aperiodic $\mu$-preserving automorphism of a Lebesgue probability space $(X, \mathcal{A}, \mu)$ onto itself and let $\sigma$ be a totally ergodic automorphism of a Lebesgue probability space $(Y, \mathcal{B}, \nu)$. Then for any $F \in \mathcal{B}$ with $\nu(F)<1$, there is a conjugate of $\tau$ which agrees with $\sigma$ on $F$; i.e., there is an invertible measure preserving bijection

$$
\psi:(Y, \mathcal{B}, \nu) \rightarrow(X, \mathcal{A}, \mu)
$$

such that

$$
\begin{aligned}
\hat{\tau}(y) & =\psi^{-1} \tau \psi(y) \\
& =\sigma(y)
\end{aligned}
$$

for $\nu$-a.e. $y \in F$.

Halmos's Conjugacy Lemma asserts that the conjugates $\mathcal{C}(\tau)$ of an aperiodic automorphism are dense in the totally ergodic automorphisms (actually dense in all of the automorphisms) of $(X, \mathcal{A}, \mu)$ if the distance between two automorphisms of $X, \tau$ and $\sigma$, is given by $\mu\{x \in X: \tau(x) \neq \sigma(x)\}$. Halmos used his Conjugacy Lemma to prove that weak mixing automorphisms are generic in the space of automorphisms with the weak topology; see [13]. Related to this, 25 years later, Katok 
and Stepin proved [16] that weak mixing volume preserving homeomorphisms are uniform topology generic in the volume preserving homeomorphisms of $X$ (when $X$ is a compact connected manifold of dimension 2 or higher).

Note however that ACT says more than Halmos's Conjugacy Lemma: namely, that we can specify in advance the set $F$, where the conjugate of $\tau$ agrees with $\sigma$ (as long as $F$ does not have full measure). This strengthening implies that the conjugates $\mathcal{C}(\tau)$ of an aperiodic automorphism are dense in the automorphisms of a compact connected manifold $X$ with the uniform convergence topology [5]. We use the ACT to show that weak topology generic properties for automorphisms are uniform topology generic properties for measure preserving homeomorphisms [5].

The previous paragraph illustrates that in approximation problems in ergodic theory, we can use the denseness of $\mathcal{C}(\tau)$ to show that if a particular aperiodic automorphism $\tau$ has a certain measure theoretic property in the space of automorphisms, then there is a dense class of automorphisms possessing the same property, namely its conjugacy class $\mathcal{C}(\tau)$, in the group of all automorphisms with the weak topology. For infinite measure spaces, a version of ACT is in [7]; see also [5]. In their memoir [1], Akin et al. define a metric space $X$ to have the Rokhlin property if there is some homeomorphism of $X$ whose conjugates (by other homeomorphisms) are dense in the space of homeomorphisms. They show that the Cantor set has the Rokhlin property.

Note that in ACT, the set $F$ is a free variable (as long as it does not have full measure). In this context, ACT is similar to Lehrer and Weiss's $\epsilon$-free Rokhlin Lemma [20, where they show that the complement of the tower of height $n$ in Rokhlin's Lemma can be chosen to be a subset of any previously prescribed measurable set. It is worth noting that for $\mathbb{Z}^{2}$-actions, an $\epsilon$-free version of the Rokhlin Lemma does not hold; see Ryzhikov [24].

We alert the reader to our use of the adjective aperiodic in these three different senses:

(1) An aperiodic automorphism $\tau$ has the property that the $\tau$-periodic points have zero measure.

(2) An aperiodic (probability) distribution $\pi=\left(\pi_{1}, \pi_{2}, \ldots\right)$ on the positive integers satisfies $\operatorname{gcd}\left\{k: \pi_{k}>0\right\}=1$.

(3) An aperiodic Markov Chain $\mathbf{P}=(p(i, j): i, j \in \mathbb{N})$ on the positive integers satisfies $\operatorname{gcd}\{n \in \mathbb{N}$ : there is some state which has period $n\}=1$.

\section{Three theorems, Four proofs, One RESUlt}

In this section we prove the equivalence of the three theorems in the previous section. We do not provide proofs of any of the three theorems; rather we refer the interested reader to our book Typical dynamics of volume preserving homeomorphisms [5] for a complete proof of MRT. We also suggest [10] for a simple proof of the special case of the MRT for finitely many columns. The equivalence of MRT, CMC and ACT follows immediately from Theorem 3.1 and theorems from [5]. However, to make this presentation self-contained, we also present a different chain of three implications to prove their equivalence.

\section{1. $\mathrm{CMC} \Rightarrow$ MRT.}

Theorem 3.1. CMC implies MRT. 
Proof. Let $\pi=\left(\pi_{1}, \pi_{2}, \ldots\right)$ be an aperiodic distribution. We seek a MultiTower $\mathcal{P}$ for $\tau$, with distribution $\pi$. Consider the following MultiTower Markov Chain (MTMC) with state space $\mathcal{T}$, the levels of the MultiTower being

$\mathcal{T}=\left\{(k, i): k\right.$ is a positive integer with $\pi_{k}>0$ and for these $\left.k, i=1,2, \ldots, k\right\}$.

On $\mathcal{T}$, we put an initial probability distribution $\mathbf{r}=\left\{r((k, i))=\pi_{k} / k:(k, i) \in \mathcal{T}\right\}$. The legal transitions of the MultiTower Markov Chain on $\mathcal{T}$ are given by: $(k, i) \rightarrow$ $\left(k^{\prime}, i^{\prime}\right)$ in $\mathcal{T}$, if $k=k^{\prime}$ and $i^{\prime}=i+1$, or $i=k$ and $i^{\prime}=1$. The nonzero transition probabilities $\mathbf{P}$ are given by the following:

$$
p((k, i),(k, i+1))=1 \text { if } i<k,
$$

and when $i=k$,

$$
p\left((k, k),\left(k^{\prime}, 1\right)\right)=\frac{r\left(\left(k^{\prime}, 1\right)\right)}{\sum_{j} r((j, 1))} .
$$

The transition probabilities on $\mathcal{T}$ with the stationary invariant probability $\mathbf{r}$ define a "mixing" Markov chain on $\mathcal{T}$ (i.e., an aperiodic, positive recurrent, irreducible Markov chain). The partition $\mathcal{R}=\left\{R_{(k, i)}:(k, i) \in \mathcal{T}\right\}$ for $\tau$, modeling the MTMC, given by the CMC Theorem is the MultiTower partition for $\tau$ having the aperiodic distribution $\pi$ that we seek; i.e., $\mathcal{P}=\left\{P_{k, i}: P_{k, i}=R_{(k, i)}\right\}$.

The implication MRT $\Rightarrow \mathbf{A C T}$ and the implication $\mathbf{A C T} \Rightarrow \mathbf{C M C}$ are from the original papers of Alpern (1981) and Alpern-Prasad (1989), or see [5]. Thus, the three theorems on multitowers, conjugacies and codes are equivalent and our main result follows.

Corollary 3.2. Let $\tau$ be an aperiodic automorphism of a Lebesgue probability space $(X, \mathcal{A}, \mu)$ onto itself. Then the three theorems for $\tau$, the Multiple Rokhlin Tower Theorem (MRT, Theorem 2.1), the Approximate Conjugacy Theorem (ACT, Theorem 2.3), and the Coding Markov Chain Theorem (CMC, Theorem 2.2), are all equivalent.

3.2. $\mathbf{M R T} \Rightarrow \mathbf{C M C}$. In order to make this presentation self-contained, we provide another proof of the previous corollary, by giving proofs of the following three implications:

- Theorem 3.3 MRT $\Rightarrow$ CMC,

- Theorem 3.4 CMC $\Rightarrow$ ACT,

- Theorem 3.5 ACT $\Rightarrow$ MRT.

Theorem 3.3, MRT $\Rightarrow$ CMC, appeared in our 1989 paper (see [5], where the latter implication follows from the chain $\mathbf{M R T} \Rightarrow \mathbf{A C T}$, and ACT $\Rightarrow \mathbf{C M C}$ ) and for the reader's convenience we include it here. The proofs of Theorems 3.4 and 3.5 have not been previously published.

Theorem 3.3. MRT implies CMC.

Proof. Let $\mathbf{P}$ be a mixing Markov Chain, which we wish to represent by $\tau$ and some partition $\mathcal{R}$ of the space. Consider the distribution $\pi=\left(\pi_{1}, \pi_{2}, \ldots\right)$, where $\pi_{k}$ is the probability that the Markov Chain starting at state 0 first returns to 0 in exactly $k$ steps. Then the aperiodicity of the Markov Chain implies that the distribution $\pi$ is aperiodic (i.e., $\operatorname{gcd}\left\{k: \pi_{k}>0\right\}=1$ ) so that MRT can be applied to $\pi$ and $\tau$. Let $\mathcal{P}=\left\{P_{k, i}: k=1,2, \ldots ; i=1,2, \ldots, k\right\}$ be a MultiTower partition of $X$ for $\tau$ with distribution $\pi$ (the distribution of columns is given by $\mu\left(\bigcup_{i=1}^{k} P_{k, i}\right)=\pi_{k}$ for each 
$k=1,2, \ldots)$. Attach the label 0 to $\bigcup_{k=1}^{\infty} P_{k, 1}$, the base of the tower. Then consider a word $w$ of length $k$ for the Markov Chain, starting at 0 and coming back to 0 for the first time in $k$ transitions, say $w=\left[0=i_{0}, i_{1}, \ldots, i_{k}=0\right]$ at 0 , and let $\alpha$ be the probability of $w$. Find a subset $F_{w}$ of the base of the $k$ th column $\left(P_{k, 1}\right)$ which occupies proportion $\alpha$ of $P_{k, 1}$. Choose the different $F_{w}$ 's (for each distinct loop $w$ for the Markov Chain) to be disjoint from each other and so that they partition the base of the MultiTower. Attach the labels $i_{1}, i_{2}, \ldots, i_{k-1}$ respectively to $\tau^{i}\left(F_{w}\right)$ for $i=1, \ldots, k-1$. When we do this for each distinct loop $w=\left[0=i_{0}, i_{1}, \ldots, i_{k}=0\right]$ at 0 , almost every point in $X$ will have a single label. Let $R_{i}$ be the set of all points with the label $i$. The partition $\mathcal{R}=\left\{R_{i}: i \in \mathbb{N}\right\}$ is the required partition which, together with $\tau$, represents the Markov Chain.

Theorems 3.3 and 3.1 together provide a proof of the equivalence of the two partition theorems CMC and MRT; this may be of some interest to probabilists.

\section{3. $\mathrm{CMC} \Rightarrow \mathrm{ACT}$.}

\section{Theorem 3.4. CMC implies ACT.}

Proof. Let $\sigma:(Y, \mathcal{B}, \nu) \rightarrow(Y, \mathcal{B}, \nu)$ be a totally ergodic automorphism of the Lebesgue probability space $(Y, \mathcal{B}, \nu)$ and let $F \in \mathcal{B}$ be any set with $\nu(F)<1$. We associate a MultiTower Markov Chain to $\sigma$ and $F$, an MTMC which captures the " $F$-orbit structure" of $\sigma$.

Since $\sigma$ is ergodic and $F$ does not have full measure, the $\sigma$-orbit of every point $y \in F$ eventually leaves $F$. For each integer $k=2,3, \ldots$, set $P_{k, 1} \subset F \backslash \sigma(F)$ to be the set of points in $F \backslash \sigma(F)$ whose $\sigma$-orbit first leaves $F$ on the $(k-1)$ th iterate. Setting $P_{k, i}=\sigma^{i-1}\left(P_{k, 1}\right)$ for $i=1,2, \ldots, k$, we note that

$$
F \cup \sigma(F)=\bigcup_{k=2}^{\infty} \bigcup_{i=1}^{k} P_{k, i}
$$

Set $P_{1,1}=Y \backslash(F \cup \sigma(F))$ and let $\mathcal{P}=\left\{P_{k, i}: k=1,2, \ldots ; i=1,2, \ldots, k\right\}$. The partition $\mathcal{P}$ provides the necessary data to construct our MTMC associated to $\sigma$ and $F$.

The MTMC $\left\{X_{n}: n=1,2, \ldots\right\}$ has as state space the MultiTower space $\mathcal{T}$,

$$
\mathcal{T}=\left\{(k, i): \nu\left(P_{k, i}\right)>0 ; k=1,2, \ldots ; i=1,2, \ldots, k\right\}
$$

with an initial probability distribution $X_{1}$ given by

$$
\mathbb{P}\left\{X_{1}=(k, i)\right\}=\nu\left(P_{k, i}\right) .
$$

The nonzero transition probabilities from a state $(k, i) \in \mathcal{T}$ are given by, for $n=$ $1,2, \ldots$,

$$
\mathbb{P}\left\{X_{n+1}=(k, i+1) \mid X_{n}=(k, i)\right\}=1 \text { if } i<k
$$

and when $i=k$,

$$
\mathbb{P}\left\{X_{n+1}=\left(k^{\prime}, 1\right) \mid X_{n}=(k, k)\right\}=\frac{\nu\left(P_{k^{\prime}, 1}\right)}{\sum_{j} \nu\left(P_{j, 1}\right)} .
$$

First note that the MTMC is aperiodic. If $\nu\left(P_{1,1}\right)>0$, then the set of positive integers which are periods for some state for the Markov chain contains 1 since the state $(1,1)$ has period 1 . Otherwise, $X=F \cup \sigma(F)=\bigcup_{k=2}^{\infty} C\left(P_{k, 1}\right)$, where 
$C\left(P_{k, 1}\right)=\bigcup_{i=1}^{k} \sigma^{i-1} P_{k, 1}$ is a $\sigma$-column of height $k$. Denote by $d$ the greatest common divisor of all possible periods for the Markov Chain. If $d>1$, the set

$$
D=\bigcup_{\{k:(k, i) \in \mathcal{T}\}} \bigcup_{\{j: j \cong 0(\bmod d), j \leq k-1\}} \tau^{j}\left(P_{k, 1}\right)
$$

is a nontrivial $d$-periodic set for $\sigma$, contradicting the fact that $\sigma$ is totally ergodic and thus has only periodic sets of full measure. Thus the Markov Chain must be aperiodic.

Clearly, the Markov Chain is irreducible (all states communicate) and the chain is thus mixing Markov; apply CMC to represent the MTMC on $\mathcal{T}$ by $\tau$ and a $\mathcal{T}$-indexed partition on $X$. Let $\mathcal{R}=\left\{R_{(k, i)}:(k, i) \in \mathcal{T}\right\}$ be a partition of $X$ which represents the MultiTower Markov Chain. Note that for each $k \geq 2$ and $i=1, \ldots, k-1, \tau\left(R_{(k, i)}\right)=R_{(k, i+1)}$. Since $\nu\left(P_{k, 1}\right)=\mu\left(R_{(k, 1)}\right)$ for each $k$, there is a measure preserving bijection $\psi: \bigcup_{k} P_{k, 1} \rightarrow \bigcup_{k} R_{(k, 1)}$. Extend $\psi:(Y, \mathcal{B}, \nu) \rightarrow$ $(X, \mathcal{A}, \mu)$ by setting for $y \in P_{k, i}, \psi(y)=\tau^{i-1} \psi \sigma^{1-i}(y)$ for all $k$ and $i=1,2, \ldots, k$. Note that since $\sigma$ and $\psi^{-1} \tau \psi$ agree on $\bigcup_{k=2} \bigcup_{i=1}^{k-1} P_{k, i}$ (the set where they differ is a subset of the "top" level of each column: $\left\{P_{k, k}: k=1,2 \ldots\right\}$ ), it follows that $\sigma(y)=\psi^{-1} \tau \psi(y)$ for all $y \in F$.

3.4. ACT $\Rightarrow$ MRT. We complete the chain of implications with the following theorem.

\section{Theorem 3.5. ACT implies MRT.}

Proof. Let $\pi=\left(\pi_{1}, \pi_{2}, \ldots\right)$ be an aperiodic probability distribution for the sought after MultiTower for $\tau$; w.l.o.g., we may assume that $\pi_{1}<1$, otherwise MRT follows trivially. First we construct a probability space $(Y, \mathcal{B}, \nu)$ and a mixing automorphism $\sigma$ of $Y$ which has a MultiTower partition $\mathcal{R}$ for $\sigma$, with distribution $\pi$. Applying ACT to $\sigma$ on an appropriate subset $F \subset Y$ will allow us to use the conjugacy to copy the partition $\mathcal{R}$ to $X$. This copy of $\mathcal{R}$ will be $\tau$ 's MultiTower partition $\mathcal{P}$ of $X$, with aperiodic distribution $\pi$.

In words, $(Y, \mathcal{B}, \nu, \sigma)$ is the shift representation of the MultiTower Markov Chain corresponding to $\pi$ (as in the proof of Theorem 3.1), $\mathcal{R}$ is the time zero partition of $Y$ and $F$ is all floors of the MultiTower which are not the top of any column. We explain these terms.

Denote the levels of the MultiTower for $\pi$ by $\mathcal{T}=\left\{(k, i):\right.$ all $k \in \mathbb{N}, \pi_{k}>0 ; i=$ $1, \ldots, k\}$. We say the transition $(k, i)$ to $\left(k^{\prime}, i^{\prime}\right)$ is $\pi$-allowable if:

(1) when $i<k$, then $k^{\prime}=k$ and $i^{\prime}=i+1$, or

(2) when $i=k$, then $k^{\prime}$ can be any positive integer with $\pi_{k^{\prime}}>0$ and $i^{\prime}=1$.

Let $Y$ be the subset of the two-sided sequence space $\mathcal{T}^{\infty}$ consisting of all $\pi$-allowable sequences of levels of the $\pi$-MultiTower; that is

$Y=\left\{\left(y_{j}\right)_{-\infty}^{\infty} \in \mathcal{T}^{\infty}:\right.$ for each $n \in \mathbb{Z}$ the transition $y_{n}$ to $y_{n+1}$ is $\pi$-allowable $\}$.

Denote by $\sigma: Y \rightarrow Y$ the left shift map defined by

$$
\sigma\left(\left(y_{i}\right)\right)=\left(y_{i}^{\prime}\right) \text { where for each } i, y_{i}^{\prime}=y_{i+1} .
$$

Consider the "time zero partition" $\mathcal{R}=\left\{R_{(k, i)}:(k, i) \in \mathcal{T}\right\}$, where

$$
R_{(k, i)}=\left\{y \in Y: y_{0}=(k, i)\right\} .
$$

We define the transition probabilities $p\left((k, i),\left(k^{\prime}, i^{\prime}\right)\right)$ corresponding to the $\pi$-allowable transitions by: 
(1) When $i<k$, then for $k^{\prime}=k$ and $i^{\prime}=i+1$, we define

$$
p((k, i),(k, i+1))=1 .
$$

(2) When $i=k$, then for each $k^{\prime}$,

$$
p\left((k, k),\left(k^{\prime}, 1\right)\right)=\frac{\pi_{k}^{\prime} / k^{\prime}}{\sum_{j}\left(\pi_{j} / j\right)} .
$$

On the product sigma algebra $\mathcal{B}$, consider the shift-invariant measure $\nu$ defined on cylinders as follows.

- Set $\nu$ on $\mathcal{R}$ by

$$
\nu\left(R_{(k, i)}\right)=\frac{\pi_{k}}{k} .
$$

- For the basic "time zero cylinder" in $\mathcal{B}$, define $\nu\left(\left\{y \in Y: y_{0}=t_{0}, y_{1}=\right.\right.$ $\left.\left.t_{1}, \ldots, y_{n}=t_{n} ; t_{j} \in \mathcal{T}\right\}\right)$ by

$$
\nu\left(R_{t_{0}} \cap \sigma^{-1} R_{t_{1}} \cap \cdots \cap \sigma^{-n} R_{t_{n}}\right)=\nu\left(R_{t_{0}}\right) \Pi_{j=1}^{n} p\left(t_{j-1}, t_{j}\right) .
$$

- Extend $\nu$ to all cylinder sets so that it is a shift-invariant measure:

$$
\begin{aligned}
\nu\left(\left\{y \in Y: y_{k}=t_{0}, y_{k+1}=\right.\right. & \left.\left.t_{1}, \ldots, y_{k+n}=t_{n} ; t_{j} \in \mathcal{T}\right\}\right) \\
& =\nu\left(\left\{y \in Y: y_{0}=t_{0}, y_{1}=t_{1}, \ldots, y_{n}=t_{n} ; t_{j} \in \mathcal{T}\right\}\right) .
\end{aligned}
$$

With this measure, the quadruple $(Y, \mathcal{B}, \nu, \sigma)$ is a mixing automorphism (the latter is straightforward, but it is a deep result of Friedman and Ornstein that $\sigma$, the shift transformation representing a mixing Markov Chain on $\mathcal{T}$, is actually a Bernoulli automorphism on $Y$ ). The partition $\mathcal{R}$ is a MultiTower for $\sigma$ with distribution $\pi$. Consider the set $F \subset Y$, defined by

$$
F=\bigcup_{k>1} \bigcup_{i=1}^{k-1} R_{(k, i)}
$$

We note that as long as $\pi_{1}<1$, then $\nu(F)<1$. Furthermore, we note that

$$
\sigma(F)=\bigcup_{k>1} \bigcup_{i=2}^{k} R_{(k, i)}=\bigcup_{k>1} \bigcup_{i=1}^{k-1} \sigma^{i}\left(R_{(k, 1)}\right)
$$

and that $Y=R_{(1,1)} \cup F \cup \sigma(F)$. We apply ACT to find a conjugate $\psi: Y \rightarrow X$ so that $\psi^{-1} \tau \psi(y)=\sigma(y)$ for all $y \in F$. Then the $\psi$-image of the partition $\mathcal{R}$ gives the following partition of $X$ :

$$
\mathcal{P}=\left\{P_{k, i}=\psi\left(R_{(k, i)}\right):(k, i) \in \mathcal{T}\right\} .
$$

For each positive integer $k \geq 2$ and $i<k$, we note that since $\sigma$ and $\psi^{-1} \tau \psi$ agree on $R_{(k, i)}$, then

$$
\tau\left(P_{k, i}\right)=P_{k, i+1} .
$$

Since the above is true for all $k \neq 1$, then $P_{1,1}$ is the column of height 1 for $\tau$ with measure $\pi_{1}$. Thus $\mathcal{P}$ is the required MultiTower partition of $X$, with distribution $\pi$. This completes our proof of the corollary. 


\section{REFERENCES}

[1] Akin, E; Hurley, M.; Kennedy, J., Dynamics of topologically generic homeomorphisms, Memoirs of the American Mathematical Society, 164 (2003), no. 783, viii+130 pp. MR1980335 (2004j:37024)

[2] Alpern, S., Rotational representations of stochastic matrices, Ann. Probab., 11 (1983), 3, 789-794. MR0704568 (85a:15022)

[3] Alpern, S.; Choksi, J. R.; Prasad, V. S., Conjugates of infinite measure preserving transformations. Canad. J. Math., 40 (1988), no. 3, 742-749. MR0960604 (89m:28026)

[4] Alpern, S.; Prasad, V. S., Return times for nonsingular measurable transformations. J. Math. Anal. Appl., 152 (1990), no. 2, 470-487. MR1077941(91m:28023)

[5] Alpern, S.; Prasad, V. S., Typical dynamics of volume preserving homeomorphisms. Cambridge Tracts in Mathematics, 139. Cambridge University Press, Cambridge, 2000. $\mathrm{xx}+216$ pp. MR 1826331 (2002i:37006)

[6] Alpern, S.; Prasad, V. S., Rotational (and other) representations of stochastic matrices. Journal of Stochastic Analysis and Applications, 26(2008), 1-15.

[7] Choksi, J. R.; Kakutani, S., Residuality of ergodic measurable transformations and of ergodic transformations which preserve an infinite measure. Indiana Univ. Math. J., 28 (1979), no. 3, 453-469. MR0529678 (80d:28042)

[8] Cohen, J. E., A geometric representation of a stochastic matrix: theorem and conjecture, The Annals of Probability, 9 (1981), 5, 899-901. MR0628884 (82j:15018)

[9] Eigen, S. J.; Hajian, A. B.; Prasad, V. S., Universal skyscraper templates for infinite measure preserving transformations, Discrete and Continuous Dynamical Systems, 16 (2006), 343360. MR2226484 (2007g:28015)

[10] Eigen, S. J.; Prasad, V. S., Multiple Rokhlin tower theorem: a simple proof, New York J. Math. 3A (1997/98), Proceedings of the New York Journal of Mathematics Conference, June 9-13, 1997, 11-14 (electronic: http://nyjm.albany.edu/8000/j/1997/3A-2.pdf). MR:1604573 (99h:28032)

[11] Grillenberger, C.; Krengel, U., On marginal distributions and isomorphisms of stationary processes, Math. Z., 149 (1976), 131-154. MR0407237(53:11020)

[12] Haigh, J., Rotational representations of stochastic matrices, Ann. Probab., 13 (1985), 3, 1024-1027. MR0799440 (87a:15031)

[13] Halmos, P. R. Lectures on ergodic theory, Chelsea Publishing Co., New York, 1960, vii+101, MR0111817 (22:677) originally published by Publications of the Mathematical Society of Japan, no. 3, The Mathematical Society of Japan, 1956, vii+99. MR0097489 (20:3958)

[14] Kalpazidou, S.; Tzouvaras, L., The forward and backward rotational decompositions of Markov chains, Stochastic Anal. Appl., 19 (2001), 3, 399-412. MR.1841536 (2002e:60110)

[15] Kalpazidou, S., Cycle representations of Markov processes: An application to rotational partitions, Stochastic processes and related topics, in Trends Math., 253-273, Birkhäuser Boston, Boston, MA, 1998. MR.1652376 (99i:60138)

[16] Katok, A. B.; Stepin, A. M., Metric properties of homeomorphisms that preserve measure, Uspehi Mat. Nauk, 25 (1970), 193-220. MR.0260974 (41:5594)

[17] Kieffer, J. C., On coding a stationary process to achieve a given marginal distribution, Ann. Probab., 8 (1980), 131-141. MR0556419 (81d:28021)

[18] Kornfeld, I., Some old and new Rokhlin towers, Contemporary Mathematics, 356, Amer. Math. Soc., Providence, RI, 2004, 145-169. MR2087594 (2005f:37006)

[19] Kornfeld, I.; Ormes, N., Topological realizations of families of automorphisms, MultiTowers and orbit equivalence, preprint.

[20] Lehrer, E.; Weiss, B., An $\varepsilon$-free Rohlin lemma, Ergodic Theory Dynamical Systems, 2 (1982), 45-48. MR 0684243 (84e:28021)

[21] Pivato, M., Building a stationary stochastic process from a finite-dimensional marginal, Canad. J. Math., 53 (2001), 382-413. MR.1820914(2002b:37009)

[22] Prikhod'ko, A. A., Partitions of the phase space of a measure-preserving $\mathbf{Z}^{\mathbf{d}}$-action into towers, Mat. Zametki, 65 (1999), 5, 712-725. MR.1716239(2001a:37007)

[23] Rudolph, D. J., A two-valued step coding for ergodic flows, Mathematische Zeitschrift, 150 (1976), 201-220. MR0414825 (54:2917) 
[24] Ryzhikov, V. V., The Rohlin-Halmos property without $\epsilon$ does not hold for the actions of the group $\mathbf{Z}^{2}$, Mat. Zametki, 44 (1988), 2, 208-215; translation in Math. Notes, 44 (1988), no. 1-2, 596-600 (1989). MR.969270 (90k:28037)

[25] Sahin, A., The $\mathbb{Z}^{d}$ Alpern Multi-Tower theorem for rectangles: A tiling approach, preprint (2007).

Delft Institute of Applied Mathematics, P. O. Box 5031, 2600 GA Delft, Netherlands Current address: London School of Economics, London WC2A 2AE, United Kingdom

E-mail address: s.alpern@lse.ac.uk

Department of Mathematics, University of Massachusetts Lowell, One University Avenue, Lowell, Massachusetts 01854

E-mail address: vidhu_prasad@uml.edu 